\title{
The Bonn Electron Stretcher Accelerator ELSA: Past and future
}

\author{
W. Hillert ${ }^{\mathrm{a}}$ \\ Physikalisches Institut, Universität Bonn, Nussallee 12, 53115 Bonn, Germany \\ / \\ Published online: 16 May 2006 - C Società Italiana di Fisica / Springer-Verlag 2006
}

\begin{abstract}
In 1953, it was decided to build a $500 \mathrm{MeV}$ electron synchrotron in Bonn. It came into operation 1958, being the first alternating gradient synchrotron in Europe. After five years of performing photoproduction experiments at this accelerator, a larger $2.5 \mathrm{GeV}$ electron synchrotron was built and set into operation in 1967. Both synchrotrons were running for particle physics experiments, until from 1982 to 1987 a third accelerator, the electron stretcher ring ELSA, was constructed and set up in a separate ring tunnel below the physics institute. ELSA came into operation in 1987, using the pulsed $2.5 \mathrm{GeV}$ synchrotron as pre-accelerator. ELSA serves either as storage ring producing synchrotron radiation, or as post-accelerator and pulse stretcher. Applying a slow extraction close to a third integer resonance, external electron beams with energies up to $3.5 \mathrm{GeV}$ and high duty factors are delivered to hadron physics experiments. Various photo- and electroproduction experiments, utilising the experimental set-ups PHOENICS, ELAN, SAPHIR, GDH and Crystal Barrel have been carried out. During the late 90's, a pulsed GaAs source of polarised electrons was constructed and set up at the accelerator. ELSA was upgraded in order to accelerate polarised electrons, compensating for depolarising resonances by applying the methods of fast tune jumping and harmonic closed orbit correction. With the experimental investigation of the GDH sum rule, the first experiment requiring a polarised beam and a polarised target was successfully performed at the accelerator. In the near future, the stretcher ring will be further upgraded to increase polarisation and current of the external electron beams. In addition, the aspects of an increase of the maximum energy to $5 \mathrm{GeV}$ using superconducting resonators will be investigated.
\end{abstract}

PACS. 29.20.-c Cyclic accelerators and storage rings - 29.20.Lq Synchrotrons - 29.27.Hj Polarised beams

\section{The beginning}

Accelerator physics started at Bonn in 1952, when Wolfgang Paul, who received the Nobel price 1989 for his work on ion traps, accepted a call to Bonn and became a full professor at the physics institute of Bonn university. Paul, who had worked at the university of Göttingen on a $6 \mathrm{MeV}$ betatron together with his teacher Hans Kopfermann, was deeply impressed by the recently discovered principle of strong focusing in particle accelerators, applying transverse magnetic fields with strong alternating field gradient $[1,2,3]$. He was thinking about building a strong focusing $100 \mathrm{MeV}$ electron synchrotron at Bonn and made an application to the Deutsche Forschungsgemeinschaft (DFG). Although this application covered about $10 \%$ of the total annual funding of the DFG, it was accepted, and Paul was even encouraged to increase the energy of the planned machine to $500 \mathrm{MeV}$.

\footnotetext{
a Since 2001 in charge of the ELSA accelerator; e-mail: hillert@physik.uni-bonn.de
}

\section{The $500 \mathrm{MeV}$ synchrotron}

In November 1953, Paul started, together with a small group of scientific assistants, PhD and diploma students, to work on the design and construction of the accelerator. At this time, there was no experience in building a strong focusing accelerator, and a lot of problems had to be overcome. Since at that time no electronic computer was available, a pendulum as a mechanical analogue was built in order to solve the differential equations describing the particles' motion. A field gradient of $10 \mathrm{~T} / \mathrm{m}$ at the maximum energy of $500 \mathrm{MeV}$ was proposed, actually the highest gradient ever used for a combined function synchrotron. After construction had started in 1954 and all of the nine magnets of type $1 / 2 D-F-1 / 2 D$ had already been installed, it turned out that the accelerator would operate just on top of a non-linear stop band, which had been discovered 1956 at CERN, and would never work. This problem could be solved by enlarging the gap between the defocusing and focusing sectors of the magnets, using $1 \mathrm{~cm}$ thick pieces of plywood. The complete radio frequency system, consisting of the transmitter, the amplifiers and the six accelerating cavities, was built in the framework of a diploma thesis [4]. Pure ceramics was used for the vacuum chamber, which 


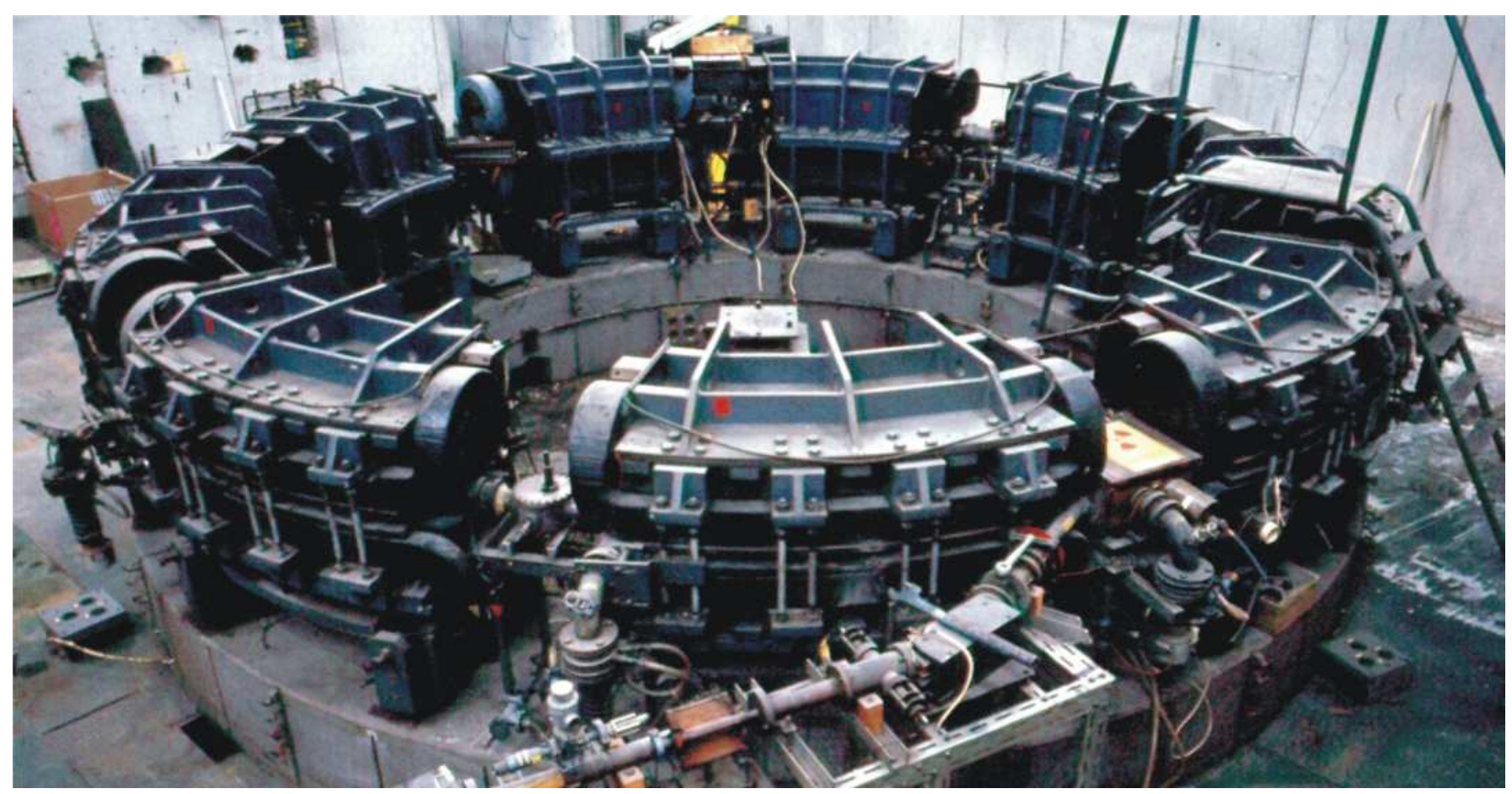

Fig. 1. The Bonn $500 \mathrm{MeV}$ strong focusing electron synchrotron.

was constructed from different tubes glued together with Araldit $^{\mathrm{TM}}$. A van de Graaff accelerator generated a $3 \mathrm{MeV}$ electron beam, which was injected into the synchrotron. The transfer beamline was calculated and set up by a $\mathrm{PhD}$ student [5], and a $60 \mathrm{kV}$ electrostatic deflector served for particle injection. Its housing was finally completely built out of Araldit ${ }^{\mathrm{TM}}$ in order to overcome the beam deteriorating effects of eddy currents, generated by the fringe fields of the bending magnets.

Figure 1 shows a photograph of the $500 \mathrm{MeV}$ synchrotron and part of its injection beamline. The main parameters of the machine are given in table 1 (see also [6]).

After overcoming a couple of additional throwbacks, the accelerator came into operation in 1958, being the first strong focusing synchrotron operational in Europe. This happened to some extent unexpectedly, and actually the institute was not prepared to perform scientific experiments at the accelerator at this time. So Paul sent a member of his machine group to the USA in order to learn about physics experiments at the weak focusing $1 \mathrm{GeV}$ synchrotron of the California Institute of Technology. In the following years, a number of pilot experiments, mainly on the photoproduction of pions off protons and deuterons, were carried out. For the first time the recoil neutron polarisation was measured. Two rotating targets produced external photon beams by the process of bremsstrahlung. The energy of the external photon beam was measured by means of tagging counters using one bending magnet of the synchrotron as dispersive element to determine the momentum of the scattered electrons.

The $500 \mathrm{MeV}$ synchrotron was operated until 1984. The total operation time exceeded 100000 hours. More than 150 diploma and doctoral theses were carried out
Table 1. Main parameters of the $500 \mathrm{MeV}$ synchrotron.
Focusing type

Number of basic periods

Basic lattice

Maximum field at design orbit

Maximum field gradient

Repetition frequency

Circumference

Orbit radius

Revolution frequency

No. of betatron oscillations per turn

Gap height at orbit

Momentum compaction factor

Coils, no. of turns per magnet unit

$\mathrm{AC}$ voltage / current

DC voltage / current

Accelerating frequency

Peak voltage per turn

Number of accelerating cavities

Injection energy

Injector type

Vacuum chamber

Pressure (4 oil diffusion pumps)
AG

9

$1 / 2 D-F-1 / 2 D$

$1 \mathrm{~T}$

$10 \mathrm{~T} / \mathrm{m}$

$50 \mathrm{~Hz}$

$16.45 \mathrm{~m}$

$1.70 \mathrm{~m}$

$18.12 \mathrm{MHz}$

2.4

$6 \mathrm{~cm}$

0.16

98

$11670 \mathrm{~V} / 215 \mathrm{~A}$

$100 \mathrm{~V} / 180 \mathrm{~A}$

$163.1 \mathrm{MHz}$

$2.5 \mathrm{kV}$

6

$3 \mathrm{MeV}$

van de Graaff

ceramics

$10^{-6}$ Torr based on the construction of the machine and scientific experiments at this accelerator.

\section{The $2.5 \mathrm{GeV}$ synchrotron}

In 1963, it became clear to the physicists performing experiments at the $500 \mathrm{MeV}$ synchrotron that the 


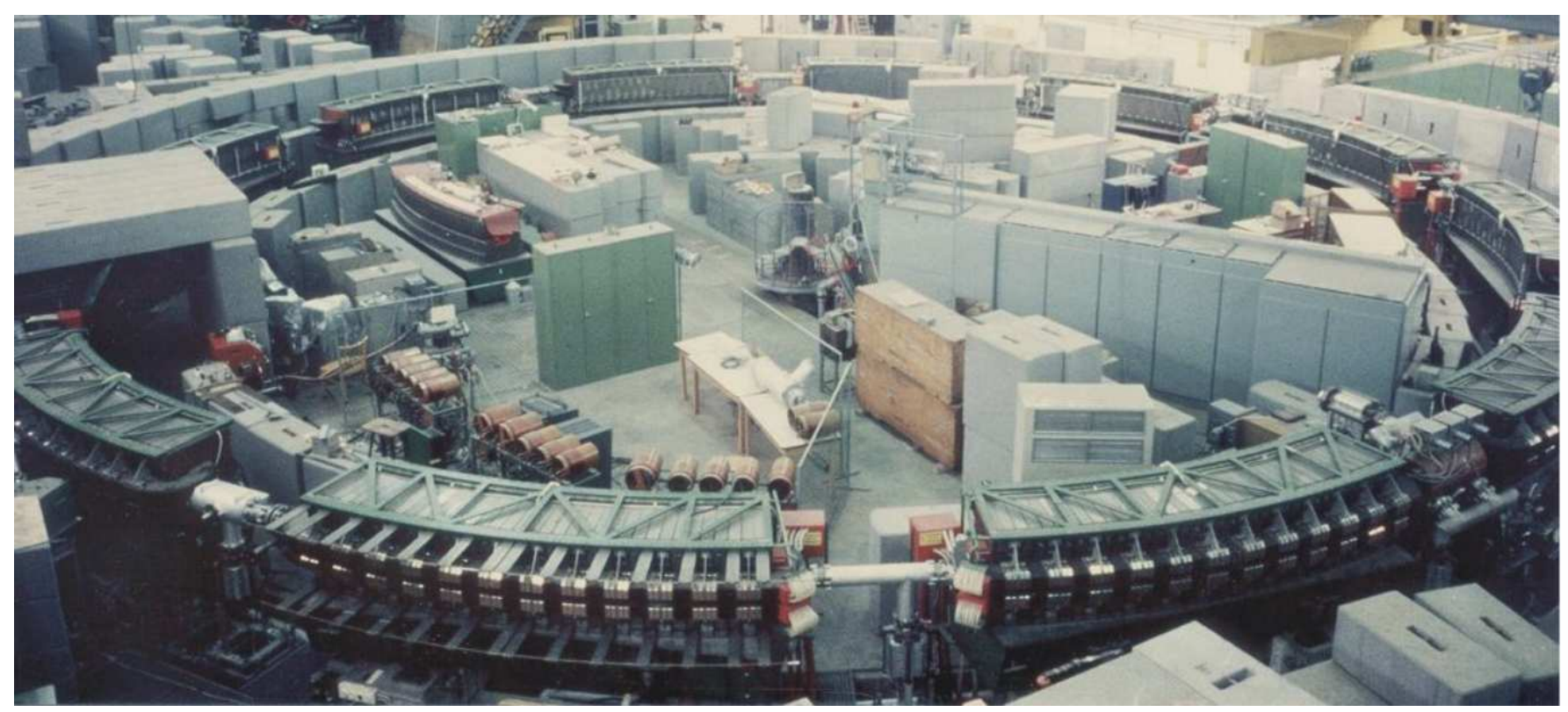

Fig. 2. The Bonn $2.5 \mathrm{GeV}$ electron synchrotron, close to its final set-up.

experimental facilities of the Bonn accelerator laboratory had to be extended in order to keep up with the development of high-energy physics worldwide. A new electron accelerator with higher energy was considered, which should again be a university machine where scientific assistants and students should participate in design and construction. At this time, the DESY synchrotron came close to operation, the Cambridge accelerator was already operating and it was decided to build the NINA synchrotron. In order to contribute to filling the gap between these $6 \mathrm{GeV}$ machines and the existing ones around $1 \mathrm{GeV}$, the Bonn group decided to build an electron synchrotron with a maximum energy of $2.5 \mathrm{GeV}$.

At the end of 1963, the state government agreed to support the new accelerator and the design of the machine started. The general dimensions of the synchrotron were fixed by the limited area of approx. $30 \times 60 \mathrm{~m}^{2}$ available between the two physics institute buildings. Therefore a simple magnetic structure with twelve combined function bending magnets based on a $O / 2-F D-O / 2$ lattice was chosen. In order to correct for chromatic effects, an additional sextupole component was introduced in the focusing and defocusing sectors of the bending magnets. To reach a short construction time the pole profile and the cross section of these AG magnets were calculated on a computer, using different two-dimensional relaxation methods which had been developed shortly before $[7,8]$. In order to avoid corrections of quadrupole and higher order fields at the time of injection, it was intended to inject at a field of at least $0.01 \mathrm{~T}$. From the beginning on a slow extraction of the electron beam was planned.

In 1964, the design was completed and the major parts were ordered. In 1965, the accelerator building and the laboratories were constructed. Manufacturing of the magnets' blocks was started based only on the performed computer simulations. In contrast to the usual design proce-
Table 2. Main parameters of the $2.5 \mathrm{GeV}$ synchrotron.

\begin{tabular}{lr}
\hline Focusing type & $\mathrm{AG}$ \\
Number of basic periods & 12 \\
Basic lattice & $O / 2-F D-O / 2$ \\
Field at $2.3 \mathrm{GeV}$ & $1.003 \mathrm{~T}$ \\
Field index $n$ & $-22.26,23.26$ \\
Repetition frequency & $50 \mathrm{~Hz}$ \\
Circumference & $69.6 \mathrm{~m}$ \\
Orbit radius & $7.65 \mathrm{~m}$ \\
Revolution frequency & $4.3074 \mathrm{MHz}$ \\
No. of betatron oscillations per turn & 3.4 \\
Gap height at orbit & $6 \mathrm{~cm}$ \\
Maximum of momentum compaction & $1.647 \mathrm{~m}$ \\
Minimum of momentum compaction & $0.81 \mathrm{~m}$ \\
Coils, no. of turns per magnet unit & 36 \\
Peak current at 1 T & $1360 \mathrm{~A}$ \\
Vacuum chamber & $\mathrm{ceramics}$ \\
Accelerating frequency & $499.67 \mathrm{MHz}$ \\
Voltage per turn & $700 \mathrm{kV}$ \\
RF peak power & $40 \mathrm{~kW}$ \\
Number of accelerating cavities & 2 \\
Injection energy & $25 \mathrm{MeV}$ \\
Injector type & linac \\
Accelerating frequency (injector) & $2998 \mathrm{MHz}$ \\
\hline &
\end{tabular}

dure at other labs, no magnet model was checked in advance. The effects of the transition zones between the Fand D-sectors and the various identical blocks of each sector, which could not be modelled by two-dimensional computer simulations, were measured after the first magnet had been assembled, and were compensated by well designed exponentially shaped end blocks forming the magnet ends.

At the end of 1966, almost all components were in place and in March 1967, the first electrons could be injected 


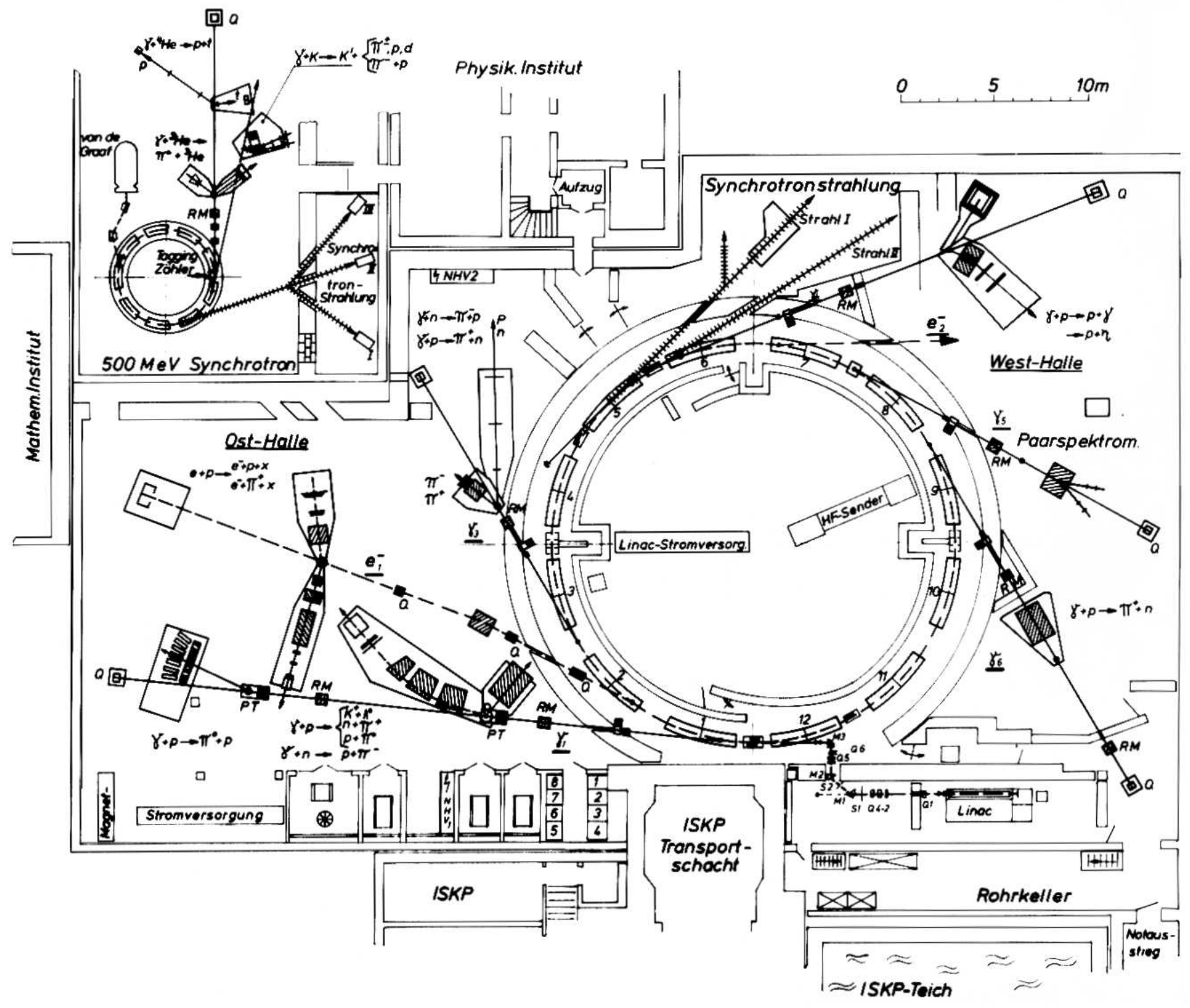

Fig. 3. Floor plan of the synchrotrons and the associated experiments.

and, without problems, accelerated up to $2.3 \mathrm{GeV}$. Acceleration was performed by means of two cylindrical RF cavity resonators, which had been developed and constructed in a special electroforming process by DESY, Hamburg [9]. A conventional television transmitter, built by Telefunken, with a modified power amplifier was used as RF power source. A linear accelerator, manufactured by Varian (Paolo Alto, California), served as injector, delivering a pulsed $25 \mathrm{MeV}$ beam with a current of $250 \mathrm{~mA}$ at an energy spread of $\pm 0.5 \%$. In July 1967, the accelerated electrons could be extracted with an efficiency of about $60 \%$. Slow beam extraction was performed by exciting a half integer horizontal betatron resonance with the help of the nonlinear magnetic field of a current strip.

The main parameters of the $2.5 \mathrm{GeV}$ synchrotron are listed in table 2 (see also [10]). Figure 2 shows a photograph of the machine, close to its final set-up. In its first operation period from 1967 to 1984, the synchrotron served as accelerator for particle physics experiments and as source for synchrotron radiation. A broad spectrum of physics experiments has been performed at this machine, starting from photo- and electroproduction of pseudoscalar mesons off protons and deuterons, continuing with photoproduction of associated strangeness in $K \Lambda / \Sigma^{0}$ and finally including the measurement of the recoil nucleon polarisation. Since 1970, polarised solid state targets (starting with polarised protons and later continuing with polarised neutrons and deuterons) became available and were also employed.

A floor plan of the experimental hall is shown in fig. 3, presenting the experimental set-ups at the two synchrotrons.

The total operation time of the $2.5 \mathrm{GeV}$ synchrotron amounted to 85000 hours for its first operation period. In 


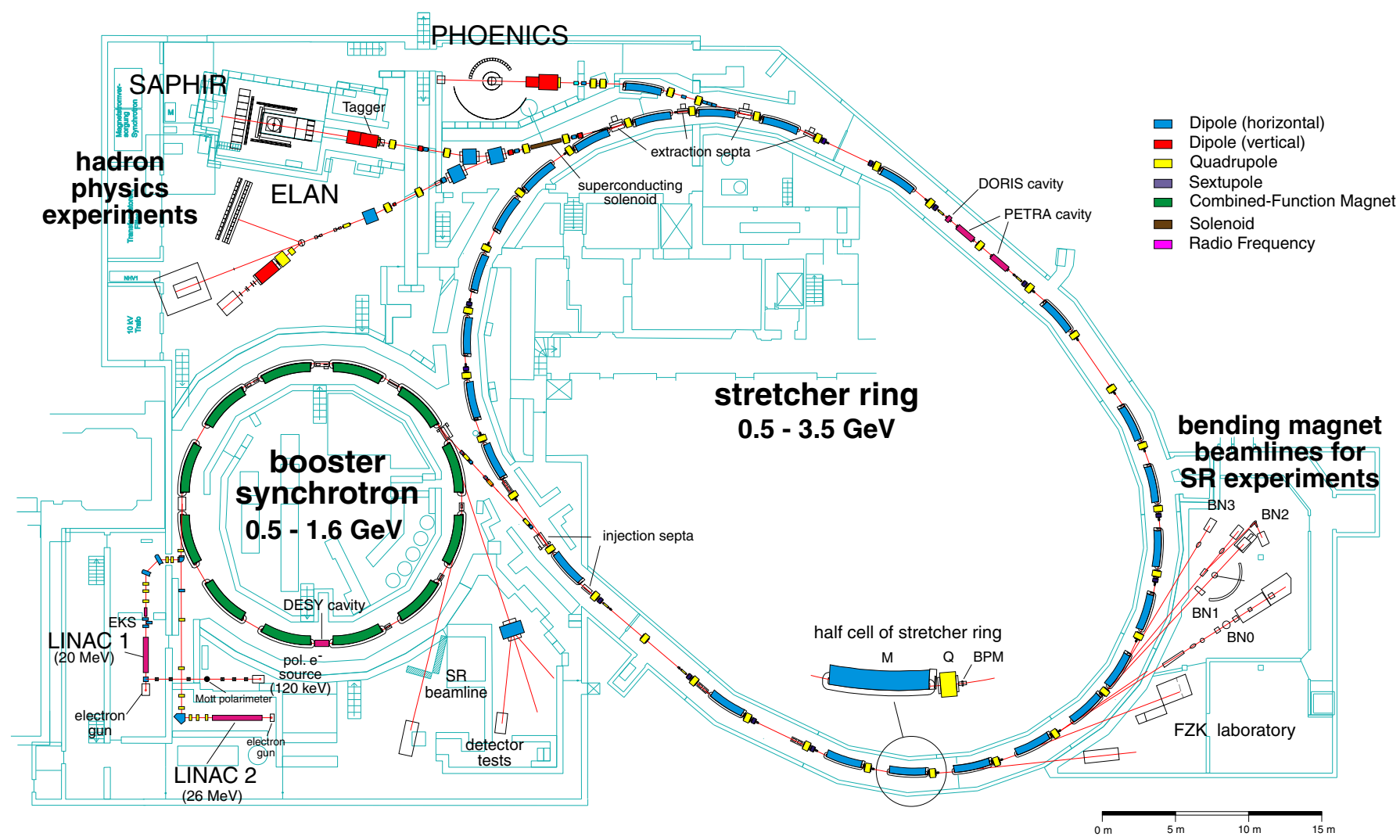

Fig. 4. Floor plan of the ELSA accelerator laboratory, indicating the first experimental set-up of the time period 1989-1997.

total 210 diploma and doctoral theses were carried out during this time.

\section{The $3.5 \mathrm{GeV}$ ELSA stretcher ring}

After more than ten years of experimental particle physics at the $2.5 \mathrm{GeV}$ synchrotron, it turned out that the quality of the experimental data was severely limited by the low duty factor of the pulsed synchrotron, which amounts to about $5 \%$ and could not be increased significantly with this type of accelerator. Following the suggestions of other laboratories around the world, it was decided to build a pulse stretcher ring, using the $2.5 \mathrm{GeV}$ synchrotron as injector [11]. A proposal was made in 1979, which was revised in 1981 and finally accepted at the end of 1981. After several years of planning and designing, the construction of the new ELectron Stretcher Accelerator ELSA started in 1982.

To avoid additional costs, the $500 \mathrm{MeV}$ synchrotron was dismantled in 1984 in order to use the existing accelerator building for experiments at the stretcher ring. The new accelerator was placed in a separate tunnel system constructed below the physics institute. ELSA is a separated function machine of simple FODO-type, which provides radiation damping of the horizontal betatron oscillations necessary for beam storage and allows a wide range variation of the betatron tune. In addition to the dipoles and quadrupoles, a total number of twelve sextupoles were installed for correction of chromatic effects and for excitation of a third integer betatron resonance needed for slow beam extraction. Two long straight sections with vanishing dispersion are equipped with the accelerating cavities and beam injection and extraction elements. Depending on the maximum beam energy chosen, a single-cell resonator of DORIS type or two five-cell resonators of PETRA type are driven by klystron-based transmitters, operating at a frequency of $500 \mathrm{MHz}$ and delivering a maximum power of $40 \mathrm{~kW}$ and $250 \mathrm{~kW}$, respectively. The vacuum system is based on thin wall $(0.3 \mathrm{~mm})$ oval tubes of stainless steel, whose rigidity is provided by $1 \mathrm{~mm}$ thick reinforcing ribs being brazed on the tubes.

A floor plan of the ELSA facility is shown in fig. 4, representing the first experimental set-up (1989-1997). The main parameters of the stretcher ring are listed in table 3.

ELSA can be operated in three different modes: the stretcher mode, the post accelerator mode and the storage mode.

Stretcher mode: Single pulses from the booster synchrotron are injected into the stretcher ring at a maximum rate of $50 \mathrm{~Hz}$. Using a slow extraction at a third integer tune, an external electron beam of constant intensity is obtained for the time between two injections. The maximum energy is limited to $1.6 \mathrm{GeV}$ by the beam transfer from the synchrotron to the stretcher ring.

Post accelerator mode: After injection of several pulses, the accumulated electron beam is accelerated to the required energy and then extracted slowly. The maximum energy obtainable is $3.5 \mathrm{GeV}$, limited by the dipole magnet 
Table 3. Main parameters of the $3.5 \mathrm{GeV}$ stretcher ring ELSA.

\begin{tabular}{lr}
\hline Focusing type & $\mathrm{AG}$ \\
Number of basic periods & 16 \\
Basic lattice & $F O D O$ \\
Field at 3.5 GeV & $1.073 \mathrm{~T}$ \\
Circumference & $164.4 \mathrm{~m}$ \\
Orbit radius & $10.88 \mathrm{~m}$ \\
Revolution frequency & $1.82 \mathrm{MHz}$ \\
Number of dipoles & 24 \\
Number of quadrupoles & 32 \\
Number of sextupoles & 12 \\
Gap height (dipole magnets) & $5 \mathrm{~cm}$ \\
Momentum compaction factor & $6.3 \%$ \\
Natural emittance (3.5 GeV) & $0.09 \%$ \\
Natural energy width (3.5 GeV) & $3015 \mathrm{~A}$ \\
Current (dipoles) at max. energy & $0.9 \mathrm{~mm} \mathrm{mrad}$ \\
Vacuum chamber & stainless steel \\
Accelerating frequency & $499.67 \mathrm{MHz}$ \\
Energy loss per turn (3.5 GeV) & $1.22 \mathrm{MeV}$ \\
Maximum RF power & $250 \mathrm{~kW}$ \\
Accelerating cavities transmitter 1 & 1 of type DORIS \\
Accelerating cavities transmitter 2 & 2 of type PETRA \\
\hline
\end{tabular}

power supply and the RF generated acceleration voltage. The "macroscopic" duty factor depends on the ramping speed and the flat top time and scales inversely proportional to the external current. For typical operation parameters $(3.2 \mathrm{GeV}, 1 \mathrm{nA}$ external current) a duty factor of about $60-70 \%$ is achieved.

Storage mode: When ELSA is operated as a synchrotron radiation source, a large number of pulses from the synchrotron are accumulated in ELSA. Then the energy is ramped slowly to the desired value and the beam is stored for hours. The beam lifetime depends on the circulating beam current and energy and amounts to 1-2 hours for typical operation parameters $(2.3 \mathrm{GeV}, 50 \mathrm{~mA})$.

Until 1994, the first set-up of the accelerator control and timing system did not allow for a fast ramping of the dipole magnets. ELSA was only operated in the stretcher and storage modes [12].

In 1990, experiments utilising the synchrotron radiation, which is emitted from the electrons when passing the bending dipole magnets of ELSA, started in a separate laboratory constructed close to the accelerator tunnel. Since that time, a total number of six bending magnet beamlines are used for X-ray lithography and radiation chemistry [13], molecular fragmentation and Auger- [14], photoemission- and X-ray absorption spectroscopy experiments [15,16] and time resolved studies [17].

For the first years, one method of beam extraction was based on scattering the circulating electrons off a carbon wire and collimating the external beam to the required beam dimensions. Since 1991, after suppressing the ripple of the main magnet power supplies by more than a factor of 100 with the help of active filters, a slow resonance extraction is successfully applied, using the following technique:

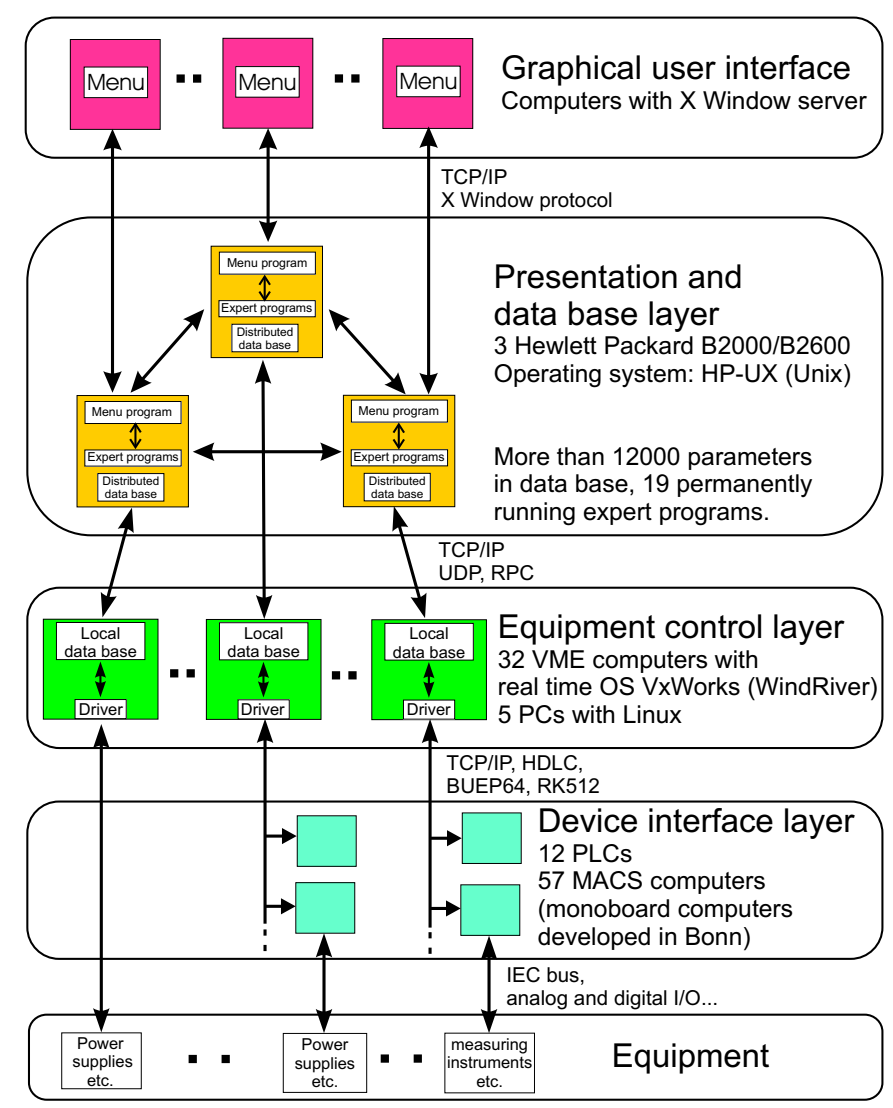

Fig. 5. Hierarchical structure of the ELSA control system (status 2005).

A third integer resonance is driven by four extraction sextupoles, located in dispersion free sections and dividing the phase space into a stable triangle and an unstable surrounding area. With the additional help of four ironless quadrupoles, the horizontal betatron tune is slowly shifted to a value of $4 \frac{2}{3}$, thereby shrinking the area of the stable triangle. When applying this procedure, part of the electron beam becomes unstable, resulting in an increasing betatron amplitude. These unstable electrons move along the separatrix lines in phase space and are finally extracted after crossing the septa of two extraction magnets.

The major problem of the pure stretcher mode operation turned out to be the achievement of a homogenous filling of ELSA with one injection from the synchrotron. Due to the circumference ratio of $3: 7$ of these two machines, a sophisticated three turn shaving extraction from the synchrotron had to be employed in order to obtain a complete filling of ELSA. Applying this method, the phase space structure of the ELSA beam often varied along the closed orbit shortly after injection, even in case of careful adjustments. This structure was not equalized by radiation damping within the short extraction time and caused small scale intensity variations of the external beam, thus decreasing the duty factor.

In 1994, a newly developed accelerator control system $[18,19]$ was set up. This system is based on three Unix workstations and in total consists of more than 100 computers (status 2005) at different layers (see fig. 5). Its 
graphical user interface combines machine steering, diagnostics and data analysis in one integrated environment. In combination with the control system, a new accelerator timing system, based on programmable delay units, was also set up. The improved systems were the key to precise and flexible tracking of the main magnets on the energy ramp and enabled a fast ramping operation (maximum speed about $7 \mathrm{GeV} / \mathrm{s}$ ) of ELSA. In addition they allow for joining of several injections from the synchrotron in ELSA with adjustable overlap, thus making a nearly perfect homogeneous filling of the stretcher ring possible. Since the successful implementation of the post accelerator mode at the end of 1994, ELSA was no longer operated in the stretcher mode.

Different experimental set-ups were supplied with an external electron beam.

The PHOENICS experiment was performed until the end of 1996, utilising a polarised frozen spin target and carrying out pion and eta photoproduction off protons and deuterons (see [20] and references therein).

The ELAN experiment (1988-1997) performed electroproduction and electrodisintegration experiments, detecting the scattered electrons with a magnetic spectrometer [21].

It was finished in August 1997 and followed up by an experiment requiring a beam of circularly polarised photons and a polarised proton target in order to investigate the contributions to the GDH sum rule in the energy range accessible at ELSA (see [22] and references therein). The GDH experiment was carried out in the years 2000-2002 after ELSA had been successfully upgraded for the operation with polarised electrons (see sect. 5).

The SAPHIR spectrometer was operated from 1991 to 1999, carrying out photoproduction experiments and concentrating on the detection of charged particles. A large variety of reactions has been studied with this detector, reaching from photoproduction of associated strangeness in $K \Lambda / \Sigma^{0}$ to the production of vector mesons $\omega, \phi$ and $\rho$ (see [23] and references therein).

SAPHIR was disassembled in 1999 and followed up by a new experimental set-up (CB@ELSA), based on the $4 \pi$ photon detector Crystal Barrel which had been moved in 1997 from LEAR/CERN to Bonn and equipped with an inner scintillating fiber detector for charged particle detection and triggering. During the first data-taking period of the CB@ELSA experiment from 2001 to 2003, proton, deuteron and solid state targets were used in photoproduction experiments (see [24] and references therein). A linearly polarised photon beam, produced by coherent bremsstrahlung off a goniometer-aligned diamond crystal, was set up in 2002 for this experiment and has been routinely available since then.

\section{Polarised electrons}

The production of polarised electron beams has been studied in Bonn for more than 35 years. Already in 1969, a source of polarised electrons, based on the photoionisation of polarised lithium atoms, was set up [25]. Beam intensity and polarisation were enlarged using circularly polarised UV laser light for photoionisation of unpolarised atoms. Based on this method (Fano effect), two sources of polarised electrons were built in Bonn; one operating with caesium, the other with rubidium gas $[26,27]$. At the Bonn $2.5 \mathrm{GeV}$ synchrotron, with the help of the rubidium source, polarised electrons were accelerated for the first time worldwide in a synchrotron [28].

The work on sources of polarised electrons was continued after the construction of ELSA, using the photoemission of GaAs crystals pumped with circularly polarised laserlight. After more than five years of work setting up a suitable vacuum system and a pulsed titanium sapphire laser of sufficient power, another source, based on a GaAs-AlGaAs superlattice photocathode was brought into operation in 1997 [29]. This $120 \mathrm{keV}$ source was used to investigate the effects of depolarising resonances in the stretcher ring [30]. During these studies, it turned out that reliability, life time and beam transfer efficiency of this source would be insufficient for hadron physics experiments.

The situation improved with the construction and assembling of a new source, adapted for the operation with a second linear accelerator, which had been moved from the university of Mainz to Bonn and installed at the $2.5 \mathrm{GeV}$ synchrotron. This $50 \mathrm{keV}$ source [31] is based on an inverted-geometry electron gun, operated in space charge limitation in order to suppress the spiking of the free running flashlamp-pumped titanium sapphire laser. Special care was taken in construction and set-up of the gun and the transfer beamline to reach and maintain a low base pressure $\left(10^{-11} \mathrm{mbar}\right)$ and extremely low partial pressures of poisoning gas species $\left(10^{-14}\right.$ mbar $)$ by application of differential pumping [32]. To improve the gun vacuum and consequently the lifetime of the photocathodes, heat cleaning and activation of the photocathodes are carried out in a load-lock system, which in addition allows to change crystals without breaking the vacuum of the gun (see fig. 6).

The $50 \mathrm{keV}$ source was operated from 2000 to 2003 with a Be-InGaAs/AlGaAs strained layer superlattice photocathode [33] for machine studies and the GDH experiment, emitting a peak current of $100 \mathrm{~mA}$ in rectangular $1 \mu$ s long electron pulses with about $80 \%$ beam polarisation and demonstrating photocathode lifetimes of more than 2000 hours.

To prevent depolarisation during acceleration in the circular accelerators due to spin precession around the guiding field of the dipole magnets, the electron spins, originally orientated longitudinally at the source, have to be rotated in order to point perpendicularly to the accelerator plane. This rotation is performed by a 90 degree electrostatic bend in the low energy beamline from the source to the linac. An additional Larmor precession in the linear accelerator, caused by its focusing solenoid lenses, is compensated by additional double solenoids in the injection beamline, which allow to vary the spin rotation angle while their focusing strengths remain fixed. After beam extraction out of the stretcher ring, a superconducting solenoid rotates the spin back into the accelerator 


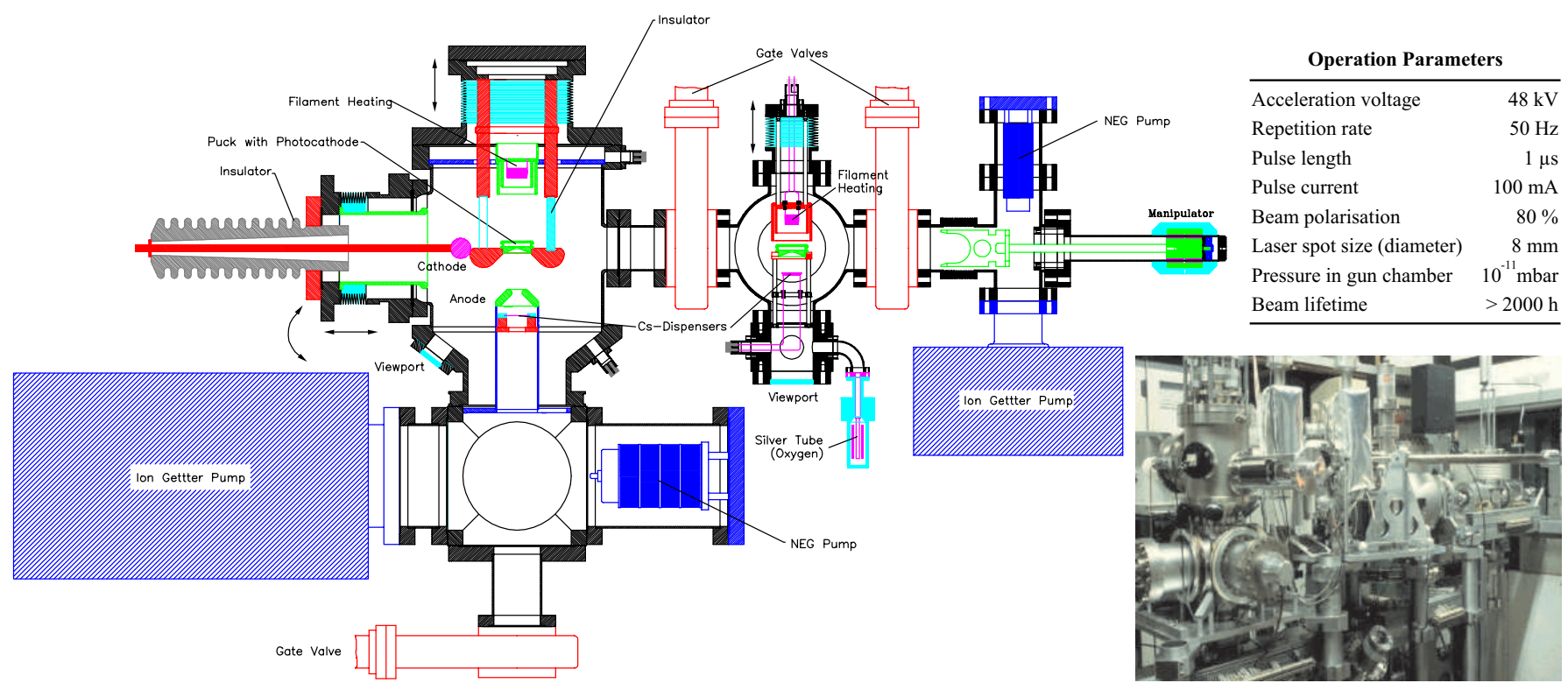

Fig. 6. Set-up of the inverted source of polarised electrons and the load-lock system.
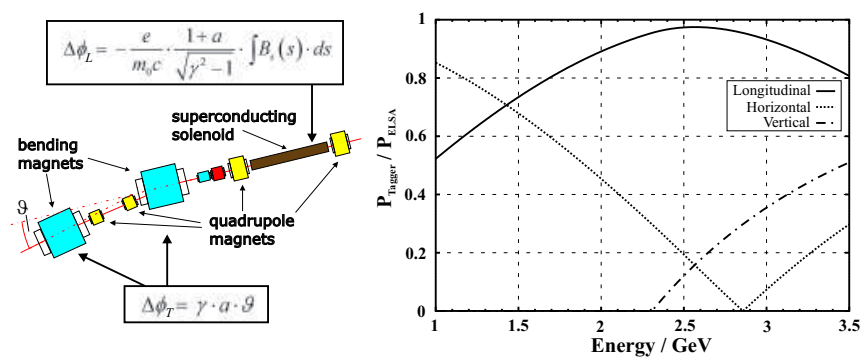

Fig. 7. Spin transfer to the tagger of the GDH experiment.

plane via Larmor precession. Longitudinal polarisation at the radiator target is obtained by Thomas precession of the spin in the bending fields of the two dipole magnets of the external beamline located downstream of the solenoid. Due to the limited field strength of the solenoid and the fixed bending angle of the dipoles, the transformation of transverse to longitudinal polarisation is incomplete and a transverse component is always present at the radiator target (see fig. 7).

Besides the incomplete spin transfer, severe beam depolarisation is caused by specific depolarising resonances appearing at certain "magic" energies in the circular accelerators. These resonances are caused by horizontal magnetic fields, present in every quadrupole and combined function bending magnet and acting on the electrons if they are passing the magnet out of its central plane. Two different types of resonances have to be distinguished:

Imperfection resonances are caused by vertical displacements of the beam or misaligned focusing elements and will depolarise the beam if the spin tune $\gamma a$ equals an integer number. In case of electrons this happens at energies which are integer multiples of $440.65 \mathrm{MeV}$.

Intrinsic resonances are caused by the finite vertical beam size originating from the vertical betatron oscillations. The resonance condition is therefore linked to

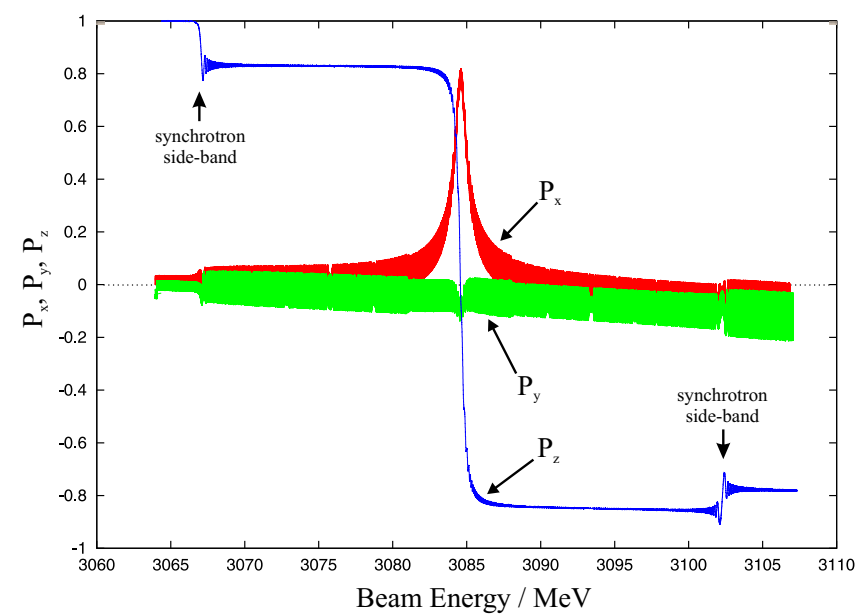

Fig. 8. Depolarisation due to crossing of synchrotron sidebands, derived from numerical simulations [35].

the machine optics and depends on the vertical betatron tune $Q_{z}$ and the superperiodicity $P$ of the accelerator: $\gamma a=k P \pm Q_{z}$, where $k$ is an integer and $P=2$ for ELSA, but $P=12$ for the booster synchrotron, respectively.

When crossing a resonance, the depolarisation $P_{f} / P_{i}$ depends on the crossing speed $\alpha$ and the resonance strength $\epsilon$. Neglecting the influence of the emission of synchrotron radiation, we obtain, applying the well known Froissart Stora formalism (see, e.g., $[34,30]$ ), a conservation of the polarisation for vanishing resonance strength and a spin flip $\left(P_{f} / P_{i}=-1\right)$ for considerably strong values of $\epsilon(|\epsilon| / \sqrt{\alpha} \gg 1)$. If additional depolarisation, originating from crossing of synchrotron side-bands, is included (see fig. 8), it turns out that a total spin flip cannot be observed at ELSA at energies higher than $1.6 \mathrm{GeV}$ [36]. Therefore, it is not feasible to enhance the strengths of all resonances sufficiently in order to avoid depolarisation, 


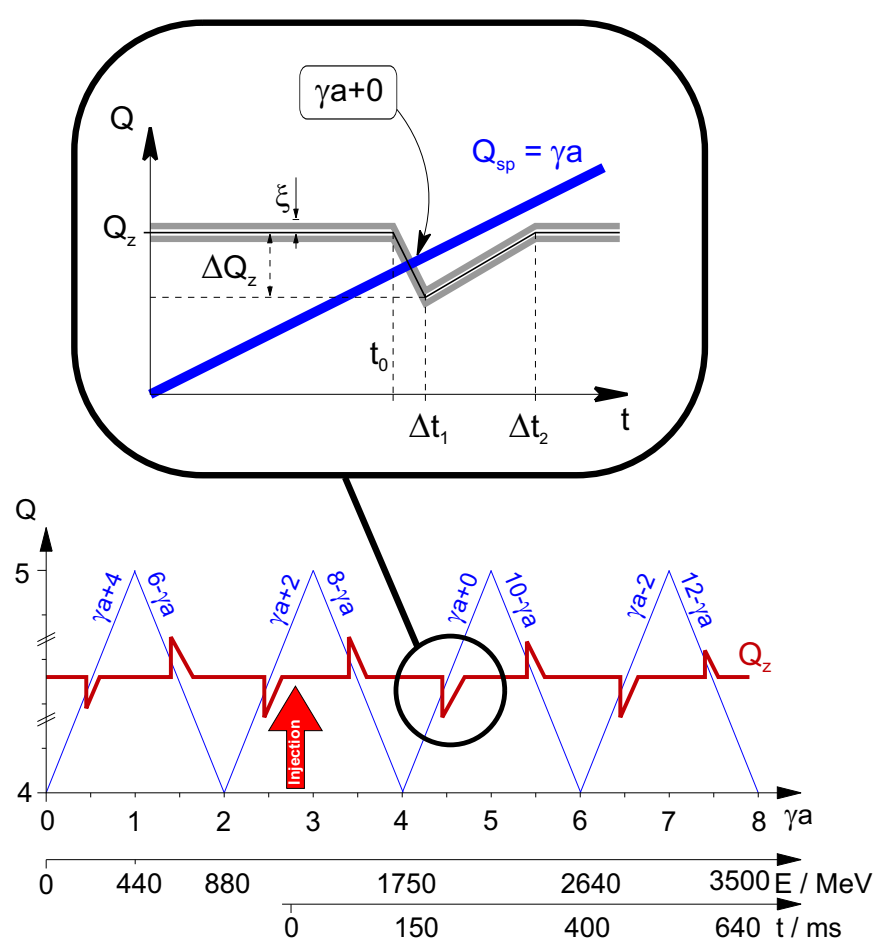

Fig. 9. Fast tune jumping of all intrinsic resonances in ELSA.

but it is required to correct for all resonances strong enough to cause a significant depolarisation.

Due to the fast ramping speed and high superperiodicity, no significant depolarisation is observed in the booster synchrotron if the beam is transferred to ELSA at energies below the third imperfection resonance at $1.32 \mathrm{GeV}$. A different situation shows up for the stretcher ring. It turned out from numerical simulations that at least nine resonances in ELSA are strong enough to produce significant depolarisation. Three techniques are applied to avoid depolarisation:

In case of intrinsic resonances, the crossing speed is enhanced with the help of two pulsed betatron tune jump quadrupoles [37], thereby shifting the vertical betatron tune by $\Delta Q_{z} \approx 0.1$ in a $\Delta t_{2} \approx 10 \mathrm{~ms}$ long triangular pulse with a rise time of $\Delta t_{1}=4 \mu \mathrm{s}$ (see fig. 9).

The strengths of the imperfection resonances are reduced with a dynamic correction of the closed orbit during the energy ramp. Vertical and horizontal displacements of the beam in the quadrupoles are measured by 28 monitor stations [38] and corrected with 19 horizontal and 21 vertical corrector magnets [39]. The following correction scheme is used: The beam is stored at the energies of the imperfection resonances and the optimal corrections are determined. Afterwards a linear interpolation between these corrections is applied during the energy ramp. The remaining distortions are generally smaller than $0.2 \mathrm{~mm}$ rms (see fig. 10).

Further reduction of the resonance strengths is achieved by correction of specific harmonics of closed orbit distortions relevant for a single imperfection resonance. This method is based on an empirical determination of two amplitude factors for each resonance by parameter
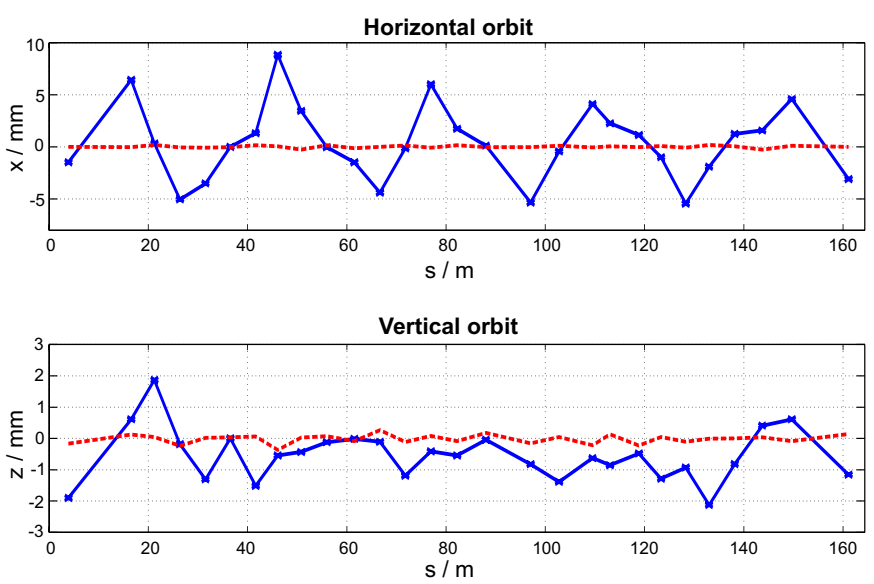

Fig. 10. Uncorrected (solid) and corrected (dashed) closed orbit.

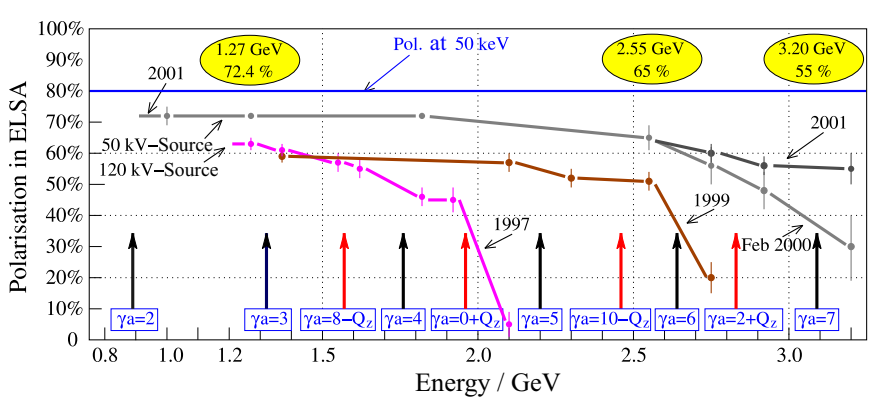

Fig. 11. Achieved beam polarisation in the stretcher ring.

variation and measuring the polarisation response of the extracted beam with a Møller polarimeter.

With all correction methods applied successfully, polarised electrons can be accelerated up to $3.2 \mathrm{GeV}$. At energies higher than $2.0 \mathrm{GeV}$ some polarisation loss is observed due to incomplete resonance compensation (see fig. 11).

\section{Future plans}

Starting in 2006, a new experimental set-up of CB@ELSA (see fig. 12) will come into operation, utilising a polarised beam and a polarised nucleon frozen spin target for photoproduction experiments in the framework of the SFB/TR 16 "Subnuclear Structure of Matter", funded by the Deutsche Forschungsgemeinschaft (DFG). With this set-up stronger demands will be put on the quality of the external electron beam. In order to enhance the beam polarisation and the external current at maximum beam energy, a number of improvements are planned in the near future, the most important of which are the following:

The 32 old quadrupole vacuum chambers will be replaced by new watercooled ones, equipped with improved monitor stations (capacitive pickups) and clearing electrodes for ion clearing.

The existing 40 corrector magnets will be removed and 60 newly designed magnets will be installed, together with four-quadrant power supplies developed at the institute. 


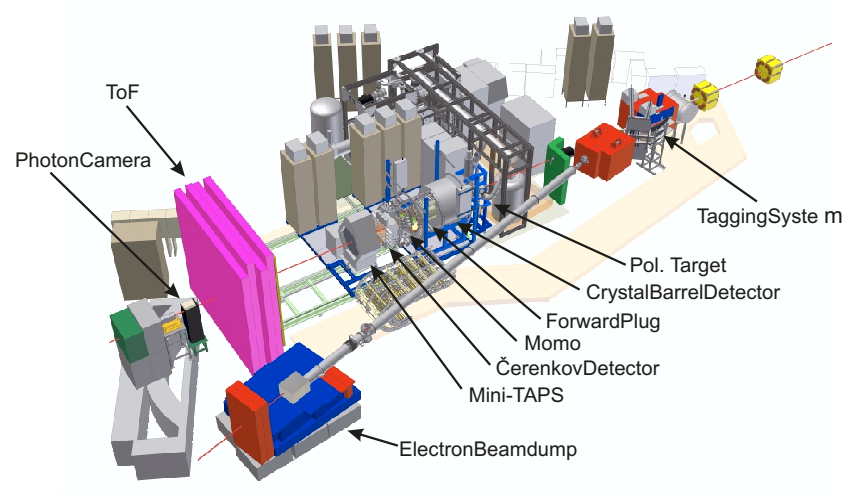

Fig. 12. New set-up of the CB@ELSA experiment.

With this correction system it will be possible to apply three times higher magnetic correction fields with a significant smaller response time which is needed for harmonic correction on the fast energy ramp at higher energies.

The first linear accelerator will be equipped with a pulsed triode gun, a subharmonic prebuncher and a TWT buncher from the disassembled SBTF set-up [40], allowing for a generation of single pulses with narrow pulse widths $(<1 \mathrm{~ns})$ and a phase synchronous operation with the synchrotron. Using this set-up a single bunch accumulation in the stretcher ring is planned, allowing time resolved measurements and detailed machine studies.

One option is to increase the maximum energy of ELSA to $5 \mathrm{GeV}$, using superconducting cavities to compensate for the considerably higher energy loss per turn of about $5.1 \mathrm{MeV}$ at $5 \mathrm{GeV}$. In order to produce the higher bending fields of $1.53 \mathrm{~T}$, the existing dipole magnets would have to be replaced by new ones with reduced gaps. The existing quadrupole and sextupole magnets will allow an operation up to $5 \mathrm{GeV}$. All the aspects of this energy upgrade will be investigated and design concepts will be worked out in near future.

I gratefully acknowledge the assistance of Professor K.H. Althoff. In many helpful discussions, he supplied me with detailed and valuable information without I could not have completed the sections on the Bonn synchrotrons. The ELSA stretcher ring was constructed and operated until 2001 under the responsibility of Professor D. Husmann. I would like to thank him and all members of the ELSA accelerator group for their contribution. Accelerator development, construction, and operation were continuously supported by DFG, BMBF, and MIWFT.

\section{References}

1. N.C. Christofilos, unpublished report (1950).

2. N.C. Christofilos, U.S. Patent no. 2.736,799, filed 1950, issued 1956.

3. E.D. Courant, M.S. Livingston, H.S. Snyder, Phys. Rev. 88, 1190-1196 (1952).

4. O. Gildemeister, diploma thesis, Bonn (1955).

5. G. Knop, doctoral thesis, Bonn (1957).
6. H. Ehrenberg, W. Paul, Die Atomwirtschaft, 300 (1959).

7. J. Drees, Proceedings of the 2nd International Conference on Magnet Technology, Oxford (1967).

8. P.F. Dahl, G. Parzen, R. Christian, IEEE Trans. Nucl. Sci. NS-12, 408 (1965).

9. H. Gerke, G. Schaffer, 5th International Congress on $\mathrm{Mi}$ crowave Tubes, Paris (1964) p. 511.

10. K.H. Althoff et al., Nucl. Instrum. Methods 61, 1-30 (1968).

11. D. Husmann et al., Proceedings of the 6th European Particle Accelerator Conference (World Scientific, Rome, 1989) p. 356.

12. K.H. Althoff et al., Part. Acc. 27, 101 (1990).

13. O. Wollersheim et al., Rad. Phys. Chem. 55, 103 (1999).

14. F. von Busch et al., Physica Scripta T 80, 401 (1999).

15. S. Bender et al., J. Non-Crystalline Solids 298, 99 (2002).

16. J. Janssen et al., Z. Anorg. Allg. Chem. 629, 1701 (2003).

17. H. Rumpf et al., J. Phys. Chem. B 105, 3415 (2001).

18. T. Götz, doctoral thesis, Bonn-IR-95-03 (1995).

19. M. Picard, doctoral thesis, Bonn-IR-95-01 (1995).

20. A. Bock et al., Phys. Rev. Lett. 81, 534 (1998).

21. H. Arenhövel et al., Phys. Rev. Lett. 75, 21 (1995).

22. H. Dutz et al., Phys. Rev. Lett. 94, 162001 (2005).

23. K.-H. Glander et al., Eur. Phys. J. A 19, 251 (2004).

24. O. Bartholomy et al., Phys. Rev. Lett. 94, 012003 (2005).

25. G. Baum, U. Koch, Nucl. Instrum. Methods 71, 189 (1969).

26. W.v. Drachenfels et al., Z. Phys. 269, 387 (1974).

27. W.v. Drachenfels et al., Nucl. Instrum. Methods 140, 47 (1977).

28. W. Brefeld et al., Nucl. Instrum. Methods A 228, 228 (1985).

29. S. Nakamura et al., 12th International Symposium on High Energy Spin Physics (World Scientific, Singapore, 1997) p. 709.

30. S. Nakamura et al., Nucl. Instrum. Methods A 411, 93 (1998).

31. W. Hillert, M. Gowin, B. Neff, AIP Conf. Proc. 570, 961 (2001).

32. W. Hillert, M. Gowin, B. Neff, Proceedings of GDH2000 (World Scientific, Singapore, 2001) p. 283.

33. T. Nakanishi et al., Proceedings of the Low Energy Polarized Electron Workshop, St. Petersburg (SPES-Publishing, St. Petersburg, 1998) p. 118.

34. M. Froissart, R. Stora, Nucl. Instrum. Methods 7, 297 (1960).

35. M. Hoffmann et al., AIP Conf. Proc. 570, 756 (2001).

36. C. Steier et al., Proceedings of the 6th European Particle Accelerator Conference, Stockholm (IOP Publishing, Bristol, 1998) p. 433.

37. C. Steier, D. Husmann, Proceedings of the 17th Particle Accelerator Conference, Vancouver (IEEE, Piscataway, 1997) p. 1033.

38. J. Dietrich, J. Keil, I. Mohos, Proceedings of the 18th Particle Accelerator Conference, New York (IEEE, Piscataway, 1999) p. 2054.

39. J. Dietrich, J. Keil, I. Mohos, Proceedings of the 4th European Workshop on Beam Diagnostics and Instrumentation for Particle Accelerators, Chester (Daresbury Laboratory, Daresbury, 1999) p. 153.

40. M. Schmitz, A.D. Yeremian, Proceedings of the 1994 International Linac Conference, Tsukuba (IEEE, Piscataway, 1994) p. 569. 